\title{
A Hilbert-Type Integral Inequality with Multiparameters and a Nonhomogeneous Kernel
}

\author{
Qiong Liu and Wenbing Sun \\ Department of Science and Information, Shaoyang University, Shaoyang 422000, China \\ Correspondence should be addressed to Wenbing Sun; swb0520@163.com
}

Received 13 February 2014; Accepted 15 May 2014; Published 1 June 2014

Academic Editor: Shanhe Wu

Copyright ( 2014 Q. Liu and W. Sun. This is an open access article distributed under the Creative Commons Attribution License, which permits unrestricted use, distribution, and reproduction in any medium, provided the original work is properly cited.

We first introduce $\Gamma$-function and Riemann $\zeta$-function to characterize the constant factor jointly. A Hilbert-type integral inequality with multiparameters and a nonhomogeneous kernel is given using the way of weight function and the technique of real analysis. The equivalent form is considered and its constant factors are proved to be the best possible. Some meaningful results are obtained by taking the special parameter values.

\section{Introduction}

If $\theta(x)(>0)$ is a measurable function and $\rho$ is a positive number, set the function spaces as follows:

$$
\begin{aligned}
& L^{2}(0, \infty):=\left\{h \geq 0 ;\|h\|_{2}:=\left\{\int_{0}^{\infty} h^{2}(x) d x\right\}^{1 / 2}<\infty\right\}, \\
& L_{\theta}^{\rho}(0, \infty) \\
& \quad:=\left\{h \geq 0 ;\|h\|_{\rho, \theta}:=\left\{\int_{0}^{\infty} \theta(x) h^{\rho}(x) d x\right\}^{1 / \rho}<\infty\right\} .
\end{aligned}
$$

If $f, g \in L^{2}(0, \infty),\|f\|_{2},\|g\|_{2}>0$, then we have [1]

$$
\iint_{0}^{\infty} \frac{f(x) g(y)}{x+y} d x d y<\pi\|f\|_{2}\|g\|_{2},
$$

where the constant factor $\pi$ is the best possible. Inequality (2) is the famous Hilbert integral inequality, which is important in analysis and its applications $[1,2]$. We note that the kernel of (2) is homogeneous of -1-degree, and the methods and results about Hilbert-type inequality with the negative homogeneous kernel and multiparameters are summarized systematically in the paper in [3]. In recent years, Hilbert-type inequality was researched from homogeneous kernel turn to nonhomogeneous kernel [4-10], such that two integral inequalities with the best constant factor are obtained in $[6,7]$ as follows:

$$
\iint_{0}^{\infty} e^{-x y} f(x) g(y) d x d y<\sqrt{\pi}\|f\|_{2}\|g\|_{2} .
$$

If $\varphi(x)=e^{-x}, \psi(y)=e^{-y}, f \in L_{\varphi}^{2}(0, \infty), g \in L_{\psi}^{2}(0, \infty)$, $\|f\|_{2, \varphi},\|g\|_{2, \psi}>0$, then

$$
\iint_{0}^{\infty} \frac{f(x) g(y)}{1+e^{x+y}} d x d y<\pi\|f\|_{2, \varphi}\|g\|_{2, \psi} .
$$

In this paper, a Hilbert-type integral inequality with nonhomogeneous kernel is given using the way of weight function and the technique of real analysis as follows.

$$
\text { If } \varphi(x)=x^{-3}, \psi(y)=y^{-3}, f \in L_{\varphi}^{2}(0, \infty), g \in L_{\psi}^{2}(0, \infty) \text {, }
$$
$\|f\|_{2, \varphi},\|g\|_{2, \psi}>0$, then

$$
\begin{gathered}
\iint_{0}^{\infty} e^{-x y} \operatorname{coth}(x y) f(x) g(y) d x d y \\
<\left(\frac{\pi^{2}}{4}-1\right)\|f\|_{2, \varphi}\|g\|_{2, \psi} .
\end{gathered}
$$




\section{Some Lemmas}

Lemma 1. Let $a>-1, \operatorname{Re}(s)>0$; then, the Laplace integral transform of the power function $x^{a}$ is as follows [11]:

$$
F(s)=\int_{0}^{\infty} x^{a} e^{-s x} d x=\frac{\Gamma(a+1)}{s^{a+1}}
$$

where $\Gamma(z)$ is the $\Gamma$-function $\left(\Gamma(z)=\int_{0}^{\infty} e^{-u} u^{z-1} d u(z>0)\right)$.

Lemma 2. Let $m$ be a positive integer; then, one has the summation formula [12]

$$
S=\sum_{k=1}^{\infty} \frac{1}{k^{2 m}}=\frac{2^{2 m-1} \pi^{2 m}}{(2 m) !} B_{m}
$$

and [8]

$$
\sum_{k=0}^{\infty} \frac{1}{(2 k+1)^{2 m}}=\frac{\left(2^{2 m}-1\right) \pi^{2 m} B_{m}}{2(2 m) !}
$$

where the $B_{m^{\prime} s}$ are the Bernoulli numbers, namely, $B_{1}=1 / 6$, $B_{2}=1 / 30, B_{3}=1 / 42, B_{4}=1 / 30, B_{5}=5 / 66, B_{6}=691 / 2730$, $B_{7}=7 / 6$, and so forth.

Lemma 3. If $p>1,1 / p+1 / q=1, \alpha, \beta>0$, define the weight function as follows:

$$
\begin{aligned}
& \omega(\alpha, \beta, x):=\int_{0}^{\infty} e^{-\alpha x y} \operatorname{coth}(\beta x y) \frac{y^{\beta}}{x^{p \beta / q}} d y, \quad x \in(0,+\infty), \\
& \omega(\alpha, \beta, y):=\int_{0}^{\infty} e^{-\alpha x y} \operatorname{coth}(\beta x y) \frac{x^{\beta}}{y^{q \beta / p}} d x, \quad y \in(0,+\infty)
\end{aligned}
$$

then, one has

$$
\begin{aligned}
& \omega(\alpha, \beta, x)=C(\alpha, \beta) x^{-p \beta-1}, \\
& \omega(\alpha, \beta, y)=C(\alpha, \beta) y^{-q \beta-1},
\end{aligned}
$$

where

$$
\begin{aligned}
C(\alpha, \beta)= & \frac{1}{2^{\beta} \beta^{\beta+1}} \Gamma(\beta+1) \\
& \times\left[\sum_{k=0}^{\infty} \frac{1}{(k+(\alpha / 2 \beta))^{\beta+1}}-\frac{1}{2}\left(\frac{2 \beta}{\alpha}\right)^{\beta+1}\right],
\end{aligned}
$$

particularly.

(1) When $\alpha=2 \beta$, by (11), we find that

$$
C(\alpha, \beta)=C(\beta)=\frac{1}{2^{\beta} \beta^{\beta+1}} \Gamma(\beta+1)\left[\zeta(\beta+1)-\frac{1}{2}\right],
$$

where $\zeta(x)$ is Riemann $\zeta$-function $\left(\zeta(x)=\sum_{k=1}^{\infty}\left(1 / k^{x}\right)(x>\right.$ 1)).
(2) When $\alpha=2 \beta, \beta=2 m-1(m=1,2, \ldots)$, from (8) and (12), we find that

$$
C(\alpha, \beta)=\frac{\pi^{2 m} B_{m}}{2 m(2 m-1)^{2 m}}-\frac{(2 m-1) !}{[2(2 m-1)]^{2 m}},
$$

where the $B_{m^{\prime} s}$ are the Bernoulli numbers, namely, $B_{1}=1 / 6$, $B_{2}=1 / 30, B_{3}=1 / 42, B_{4}=1 / 30, B_{5}=5 / 66, B_{6}=691 / 2730$, $B_{7}=7 / 6$, and so forth.

Proof. Setting $\beta x y=u$, then, by (6), we have

$$
\begin{aligned}
\omega(\alpha, \beta, x)= & \int_{0}^{\infty} e^{-\alpha x y} \operatorname{coth}(\beta x y) \frac{y^{\beta}}{x^{p \beta / q}} d y \\
= & \frac{1}{\beta^{\beta+1}} x^{-p \beta-1} \int_{0}^{\infty} \frac{e^{-(\alpha / \beta) u}+e^{-(2+\alpha / \beta) u}}{1-e^{-2 u}} u^{\beta} d u \\
= & \frac{1}{\beta^{\beta+1}} x^{-p \beta-1} \\
& \times \sum_{k=0}^{\infty}\left\{\frac{\Gamma(\beta+1)}{(2 k+\alpha / \beta)^{\beta+1}}+\frac{\Gamma(\beta+1)}{[2(k+1)+\alpha / \beta]^{\beta+1}}\right\} \\
= & \frac{1}{2^{\beta} \beta^{\beta+1}} x^{-p \beta-1} \Gamma(\beta+1) \\
& \times\left[\sum_{k=0}^{\infty} \frac{1}{(k+\alpha / 2 \beta)^{\beta+1}}-\frac{1}{2}\left(\frac{2 \beta}{\alpha}\right)^{\beta+1}\right] \\
= & C(\alpha, \beta) x^{-p \beta-1} .
\end{aligned}
$$

By the same way, we obtain $\omega(\alpha, \beta, y)=C(\alpha, \beta) y^{-q \beta-1}$.

Lemma 4. If $p>1,1 / p+1 / q=1, \alpha, \beta>0,0<\varepsilon<$ $\min \{q \beta, p \beta\}$, and $\varepsilon$ is small enough, define the real functions as follows:

$$
\begin{aligned}
& \tilde{f}(x)= \begin{cases}0, & x \in(0,1), \\
x^{(p \beta-\varepsilon) / p}, & x \in[1, \infty),\end{cases} \\
& \tilde{g}(y)= \begin{cases}0, & y \in(1, \infty), \\
y^{(q \beta+\varepsilon) / q}, & y \in(0,1] ;\end{cases}
\end{aligned}
$$

then, one finds that

$$
\begin{aligned}
\widetilde{J} \varepsilon= & {\left[\int_{0}^{\infty} x^{-p \beta-1} \widetilde{f}^{p}(x) d x\right]^{1 / p} } \\
& \times\left[\int_{0}^{\infty} y^{-q \beta-1} \widetilde{g}^{q}(y) d y\right]^{1 / q} \varepsilon=1, \\
\widetilde{I} \varepsilon= & \iint_{0}^{\infty} e^{-\alpha x y} \operatorname{coth}(\beta x y) \tilde{f}(x) \widetilde{g}(y) d x d y \\
> & C(\alpha, \beta)(1-o(1)) \quad\left(\varepsilon \longrightarrow 0^{+}\right) .
\end{aligned}
$$


Proof. We easily get

$$
\begin{aligned}
\widetilde{J} \varepsilon & =\left[\int_{0}^{\infty} x^{-p \beta-1} \widetilde{f}^{p}(x) d x\right]^{1 / p}\left[\int_{0}^{\infty} y^{-q \beta-1} \widetilde{g}^{q}(y) d y\right]^{1 / q} \varepsilon \\
& =\left[\int_{1}^{\infty} x^{-(1+\varepsilon)} d x\right]^{1 / p}\left[\int_{0}^{1} y^{-1+\varepsilon} d y\right]^{1 / q} \varepsilon=1 .
\end{aligned}
$$

Since $F(u)=u^{\beta+2} e^{-(\alpha / \beta) u}$ coth $u$ is continuous on $(0, \infty)$ and $\lim _{u \rightarrow 0} F(u)=0, \lim _{u \rightarrow \infty} F(u)=0$, there exists $M>0$, such that $F(u) \leq M$. From Fubini theorem [13], we have

$$
\begin{aligned}
& \widetilde{I} \varepsilon=\iint_{0}^{\infty} e^{-\alpha x y} \operatorname{coth}(\beta x y) \tilde{f}(x) \tilde{g}(y) d x d y \\
& =\varepsilon \int_{1}^{\infty} x^{(p \beta-\varepsilon) / p} d x\left[\int_{0}^{1} e^{-\alpha x y} \operatorname{coth}(\beta x y) y^{(q \beta+\varepsilon) / q} d y\right] \\
& =\frac{\varepsilon}{\beta^{\beta+1+\varepsilon / q}} \\
& \times \int_{1}^{\infty} x^{-1-\varepsilon} d x\left[\int_{0}^{\beta x} e^{-(\alpha / \beta) u}(\operatorname{coth} u) u^{\beta+\varepsilon / q} d u\right] \\
& =\frac{\varepsilon}{\beta^{\beta+1+\varepsilon / q}} \int_{0}^{\infty} u^{\beta+\varepsilon / q} e^{-(\alpha / \beta) u} \operatorname{coth} u d u \\
& -\frac{\varepsilon}{\beta^{\beta+1+\varepsilon / q}} \int_{1}^{\infty} x^{-1-\varepsilon} d x \int_{a x}^{\infty} u^{\beta+\varepsilon / q} e^{-(\alpha / \beta) u} \operatorname{coth} u d u \\
& =\frac{1}{\beta^{\beta+1+\varepsilon / q}} \Gamma\left(\beta+1+\frac{\varepsilon}{q}\right) \\
& \times \sum_{k=0}^{\infty}\left\{\frac{1}{(2 k+\alpha / \beta)^{\beta+1}}+\frac{1}{[2(k+1)+\alpha / \beta]^{\beta+1}}\right\} \\
& -\frac{1}{\beta^{\beta+1+\varepsilon / q}} \int_{1}^{\infty} x^{-1-\varepsilon} d x \int_{a x}^{\infty} u^{\beta+\varepsilon / q} e^{-(\alpha / \beta) u} \operatorname{coth} u d u \\
& >\frac{1}{2^{\beta} \beta^{\beta+1+\varepsilon / q}} \Gamma\left(\beta+1+\frac{\varepsilon}{q}\right) \\
& \times\left[\sum_{k=0}^{\infty} \frac{1}{(k+\alpha / 2 \beta)^{\beta+1}}-\frac{1}{2}\left(\frac{2 \beta}{\alpha}\right)^{\beta+1}\right] \\
& -\frac{M \varepsilon}{\beta^{\beta+1+\varepsilon / q}} \int_{1}^{\infty} x^{-1} d x \int_{a x}^{\infty} u^{-2+\varepsilon / q} d u \\
& =\frac{1}{2^{\beta} \beta^{\beta+1+\varepsilon / q}} \Gamma\left(\beta+1+\frac{\varepsilon}{q}\right) \\
& \times\left[\sum_{k=0}^{\infty} \frac{1}{(k+\alpha / 2 \beta)^{\beta+1}}-\frac{1}{2}\left(\frac{2 \beta}{\alpha}\right)^{\beta+1}\right]
\end{aligned}
$$

$$
\begin{aligned}
& -\frac{M \varepsilon}{\beta^{\beta+1}} \frac{1}{(1-\varepsilon / q)^{2}} \\
= & C(\alpha, \beta)(1-o(1)) \quad\left(\varepsilon \longrightarrow 0^{+}\right) .
\end{aligned}
$$

\section{Main Results and Applications}

Theorem 5. If $p>1,1 / p+1 / q=1, \alpha, \beta>0, \varphi(x)=x^{-p \beta-1}$, $\psi(y)=y^{-q \beta-1}, f \in L_{\varphi}^{p}(0, \infty), g \in L_{\psi}^{q}(0, \infty),\|f\|_{p, \varphi},\|g\|_{q, \psi}>$ 0 , then one has

$$
\begin{gathered}
\iint_{0}^{\infty} e^{-\alpha x y} \operatorname{coth}(\beta x y) f(x) g(y) d x d y \\
<C(\alpha, \beta)\|f\|_{p, \varphi}\|g\|_{q, \varphi},
\end{gathered}
$$

where the constant factor $C(\alpha, \beta)$ (with (11)) is the best possible.

Proof. By Hölder's inequality [14], Fubini theorem, and Lemma 4, we obtain

$$
\begin{aligned}
I:= & \iint_{0}^{\infty} e^{-\alpha x y} \operatorname{coth}(\beta x y) f(x) g(y) d x d y \\
= & \iint_{0}^{\infty} e^{-\alpha x y} \operatorname{coth}(\beta x y) f(x) g(y) \\
& \times\left[\frac{y^{\beta / p}}{x^{\beta / q}}\right]\left[\frac{x^{\beta / q}}{y^{\beta / p}}\right] d x d y \\
\leq & {\left[\iint_{0}^{\infty} e^{-\alpha x y} \operatorname{coth}(\beta x y) f^{p}(x) \frac{y^{\beta}}{x^{p / q}} d x d y\right]^{1 / p} } \\
& \times\left[\iint_{0}^{\infty} e^{-\alpha x y} \operatorname{coth}(\beta x y) g^{q}(y) \frac{x^{\beta}}{y^{q \beta / p}} d x d y\right]^{1 / q} \\
= & \left\{\int_{0}^{\infty} \omega(\alpha, \beta, x) f^{p}(x) d x\right\}^{1 / p} \\
& \times\left\{\int_{0}^{\infty} \omega(\alpha, \beta, y) g^{q}(y) d y\right\}^{1 / q} \\
= & C(\alpha, \beta)\|f\|_{p, \varphi}\|g\|_{q, \varphi}
\end{aligned}
$$

If inequality (21) keeps the form of equality, then there exist constants $A$ and $B$ [14], which are not all zeroes, such that $A\left(y^{\beta} / x^{p \beta / q}\right) f^{p}(x)=B\left(x^{\beta} / y^{q \beta / p}\right) g^{q}(y)$ a.e. in $(0, \infty) \times(0, \infty)$. It follows that $A x^{-p \beta} f^{p}(x)=B y^{-q \beta} g^{q}(y)$ a.e. on $(0, \infty) \times$ $(0, \infty)$. Assuming that $A \neq 0$, there exists $y>0$, such that $x^{-p \beta-1} f^{p}(x)=\left[B y^{-q \beta} g^{q}(y)\right](1 / A x)$ a.e. in $x \in(0, \infty)$, which contradicts the fact that $0<\|f\|_{p, \varphi}<\infty$. Then, inequality (21) keeps the strict form.

If the constant factor $C(\alpha, \beta)$ of $(20)$ is not the best possible, then there exists a positive $K<C(\alpha, \beta)$, such that inequality (20) is still valid if we replace $C(\alpha, \beta)$ by $K$; then, by (16) and (17), we have

$$
C(\alpha, \beta)(1-o(1))<K \text {. }
$$


Letting $\varepsilon \rightarrow 0^{+}$, we get $K \geq C(\alpha, \beta)$, which contradicts the fact that $K<C(\alpha, \beta)$, so the constant factor $C(\alpha, \beta)$ of $(20)$ is the best possible.

Theorem 6. If $p>1,1 / p+1 / q=1, \alpha, \beta>0, \varphi(x)=$ $x^{-p \beta-1} f \in L_{\varphi}^{p}(0, \infty),\|f\|_{p, \varphi}>0$, then one has

$$
\begin{gathered}
\int_{0}^{\infty} y^{(q \beta+1) /(q-1)} d y\left[\int_{0}^{\infty} e^{-\alpha x y} \operatorname{coth}(\beta x y) f(x) d x\right]^{p} \\
<C^{p}(\alpha, \beta)\|f\|_{p, \varphi}^{p},
\end{gathered}
$$

where the constant factor $C^{p}(\alpha, \beta)$ is the best possible, and inequality (23) is equivalent to inequality (20).

Proof. Setting a bounded measurable function as

$$
[f(x)]_{n}:=\min \{n, f(x)\}= \begin{cases}f(x), & \text { for } f(x)<n \\ n, & \text { for } f(x) \geq n\end{cases}
$$

since $0<\|f\|_{p, \varphi}<\infty$, there exists $n_{0} \in \mathbf{N}$, such that $0<$ $\int_{1 / n}^{n} \varphi_{p}(x)[f(x)]_{n}^{p} d x<\infty\left(n \geq n_{0}\right)$. Setting

$$
\begin{array}{r}
g_{n}(y):=y^{(p \beta+1) /(q-1)}\left[\int_{1 / n}^{n} e^{-\alpha x y} \operatorname{coth}(\beta x y)[f(x)]_{n} d x\right]^{p / q}, \\
\left(\frac{1}{n}<y<n, n \geq n_{0}\right),
\end{array}
$$

when $n \geq n_{0}$, from (20), we find that

$$
\begin{aligned}
0< & \int_{1 / n}^{n} \psi(y) g_{n}^{q}(y) d y \\
= & \int_{1 / n}^{n} y^{(q \beta+1) /(q-1)}\left[\int_{1 / n}^{n} e^{-\alpha x y} \operatorname{coth}(\beta x y)[f(x)]_{n} d x\right]^{p} d y \\
= & \iint_{1 / n}^{n} e^{-\alpha x y} \operatorname{coth}(\beta x y)[f(x)]_{n} g_{n}(y) d x d y \\
< & C(\alpha, \beta)\left\{\int_{1 / n}^{n} \varphi(x)[f(x)]_{n}^{p} d x\right\}^{1 / p} \\
& \times\left\{\int_{1 / n}^{n} \psi(y) g_{n}^{q}(y) d y\right\}^{1 / q}, \\
0< & \int_{1 / n}^{n} \psi(y) g_{n}^{q}(y) d y<C^{p}(\alpha, \beta) \int_{0}^{\infty} \varphi(x) f^{p}(x) d x \\
= & C^{p}(\alpha, \beta)\|f\|_{p, \varphi}^{p}<\infty .
\end{aligned}
$$

It follows that $0<\|f\|_{p, \varphi}<\infty$. For $n \rightarrow \infty$, by (20), (26) still keep the forms of strict inequality. Hence, we have (23).
On the other hand, from Hölder inequality, we find that

$$
\begin{aligned}
I= & \iint_{0}^{\infty} e^{-\alpha x y} \operatorname{coth}(\beta x y) f(x) g(y) d x d y \\
= & \int_{0}^{\infty}\left[y^{(q \beta+1) / p(q-1)} \int_{0}^{\infty} e^{-\alpha x y} \operatorname{coth}(\beta x y) f(x) d x\right] \\
& \times\left[y^{(-q \beta-1) / p(q-1)} g(y)\right] d y \\
\leq & \left\{\int_{0}^{\infty} y^{(q \beta+1) /(q-1)} d y\left[\int_{0}^{\infty} e^{-\alpha x y} \operatorname{coth}(\beta x y) f(x) d x\right]^{p}\right\}^{1 / p} \\
& \times\|g\|_{q, \varphi} \\
< & C(\alpha, \beta)\|f\|_{p, \varphi}\|g\|_{q, \varphi} .
\end{aligned}
$$

The inequality is (20), which is equivalent to (23).

If the constant factor of (23) is not the best, by (23), then we obtain that the constant factor of (20) is not the best too, which contradicts Theorem 5 . Thus, the constant factor $C^{p}(\alpha, \beta)$ in $(23)$ is the best possible.

By taking the special parameter values in (20) and (23), some meaningful inequalities are obtained as follows.

Example 7. Let $\alpha=\beta=1, p=q=2$; by (8) and (11), we get $C(1,1)=2 \sum_{k=0}^{\infty}\left(1 /(2 k+1)^{2}\right)-1=\pi^{2} / 4-1$. If $\varphi(x)=x^{-3}$, $\psi(y)=y^{-3}, f \in L_{\varphi}^{2}(0, \infty), g \in L_{\psi}^{2}(0, \infty),\|f\|_{2, \varphi},\|g\|_{2, \psi}>0$, then we have (5) and its equivalent form as

$$
\int_{0}^{\infty} y^{3} d y\left[\int_{0}^{\infty} e^{-x y} \operatorname{coth}(x y) f(x) d x\right]^{2}<\left(\frac{\pi^{2}}{4}-1\right)^{2}\|f\|_{2, \varphi}^{2}
$$

where the constant factor $\left(\pi^{2} / 4-1\right)^{2}$ is the best possible.

Example 8. Let $\alpha=1, \beta=1 / 2, p=q=2$; by (12), we get $C(1,1 / 2)=\sqrt{\pi}[\zeta(3 / 2)-1 / 2]=1.87204391^{+}$. If $\varphi(x)=x^{-2}$, $\psi(y)=y^{-2}, f \in L_{\varphi}^{2}(0, \infty), g \in L_{\psi}^{2}(0, \infty),\|f\|_{2, \varphi},\|g\|_{2, \psi}>0$, then we have the following equivalent inequalities:

$$
\begin{gathered}
\iint_{0}^{\infty} e^{-x y} \operatorname{coth}\left(\frac{1}{2} x y\right) f(x) g(y) d x d y \\
<\sqrt{\pi}\left[\zeta\left(\frac{3}{2}\right)-\frac{1}{2}\right]\|f\|_{2, \varphi}\|g\|_{2, \psi}, \\
\int_{0}^{\infty} y^{2} d y\left[\int_{0}^{\infty} e^{-x y} \operatorname{coth}\left(\frac{1}{2} x y\right) f(x) d x\right]^{2} \\
<\pi\left[\zeta\left(\frac{3}{2}\right)-\frac{1}{2}\right]^{2}\|f\|_{2, \varphi}^{2},
\end{gathered}
$$

where the constant factors $\sqrt{\pi}[\zeta(3 / 2)-1 / 2], \pi[\zeta(3 / 2)-1 / 2]^{2}$ in (29) are the best possible.

Example 9. Let $\alpha=2, \beta=1, p=q=2$; by (16), we get $C(2,1)=(1 / 4)\left(\pi^{2} / 3-1\right)$. If $\varphi(x)=x^{-3}, \psi(y)=y^{-3}$, 
$f \in L_{\varphi}^{2}(0, \infty), g \in L_{\psi}^{2}(0, \infty),\|f\|_{2, \varphi},\|g\|_{2, \psi}>0$, then we have the following equivalent inequalities:

$$
\begin{gathered}
\iint_{0}^{\infty} e^{-2 x y} \operatorname{coth}(x y) f(x) g(y) d x d y \\
<\frac{1}{4}\left(\frac{\pi^{2}}{3}-1\right)\|f\|_{2, \varphi}\|g\|_{2, \psi}, \\
\int_{0}^{\infty} y^{3} d y\left[\int_{0}^{\infty} e^{-2 x y} \operatorname{coth}(x y) f(x) d x\right]^{2} \\
<\frac{1}{16}\left(\frac{\pi^{2}}{3}-1\right)^{2}\|f\|_{2, \varphi}^{2},
\end{gathered}
$$

where the constant factors $(1 / 4)\left(\pi^{2} / 3-1\right),(1 / 16)\left(\pi^{2} / 3-1\right)^{2}$ in (30) are the best possible.

\section{Conflict of Interests}

The authors declare that there is no conflict of interests regarding the publication of this paper.

\section{Acknowledgment}

This paper was funded by the National Natural Science Foundation of China (no. 11171280).

\section{References}

[1] G. H. Hardy, J. E. Littlewood, and G. Pólya, Inequalities, Cambridge University Press, Cambridge, UK, 1952.

[2] D. S. Mitrinović, J. E. Pečarić, and A. M. Fink, Inequalities Involving Functions and their Integrals and Derivatives, vol. 53, Kluwer Academic, Boston, Mass, USA, 1991.

[3] B. C. Yang, "A survey of the study of Hilbert-type inequalities with parameters," Advances in Mathematics, vol. 38, no. 3, pp. 257-268, 2009.

[4] B. Yang, "On the norm of a Hilbert's type linear operator and applications," Journal of Mathematical Analysis and Applications, vol. 325, no. 1, pp. 529-541, 2007.

[5] J. M. Li and Q. Liu, "A generalization of Hardy-Hilbert's inequality and its applications," Acta Mathematica Sinica. Chinese Series, vol. 52, no. 2, pp. 237-244, 2009.

[6] B. Yang, "A new Hilbert-type inequality," Bulletin of the Belgian Mathematical Society, vol. 13, no. 3, pp. 479-487, 2006.

[7] B. C. Yang, "A Hilbert-type integral inequality with nonhomogeneous kernel," Journal of Shanghai University. Natural Science, vol. 17, no. 5, pp. 603-605, 2011.

[8] Q. Liu and S. C. Long, "A Hilbert-type integral inequality with kernel a hyperbolic secant function," Journal of Zhejiang University, vol. 40, no. 3, pp. 255-259, 2013.

[9] Q. Liu and B. C. Yang, "A Hilbert-type integral inequality with a mixed kernel of some parameters and its applications," Journal of Zhejiang University, vol. 39, no. 2, pp. 135-141, 2012.

[10] B. C. Yang, "An extended Hilbert-type integral inequality with a nonhomogeneous kernel," Journal of Jilin University, vol. 48, no. 5, pp. 719-722, 2010.
[11] B. Shu and D. Chen, Complex-Variable Function and Integral Transform, Higher Education Press, Beijing, China, 2003.

[12] Z. Huang and D. Guo, An Intruction to Special Function, Beijing Press, Beijing, China, 2000.

[13] J. Kuang, Introduction to Real Analysis, Hunan Edueation Press, Changsha, China, 1996.

[14] J. Kuang, Applied Inequalities, Shandong Science and Technology Press, Jinan, China, 3rd edition, 2004. 


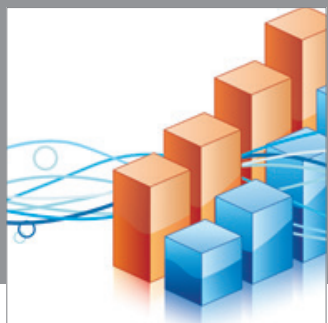

Advances in

Operations Research

mansans

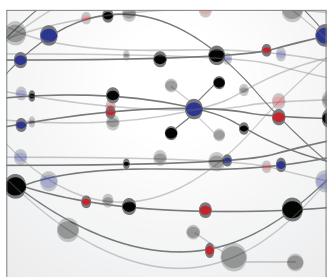

The Scientific World Journal
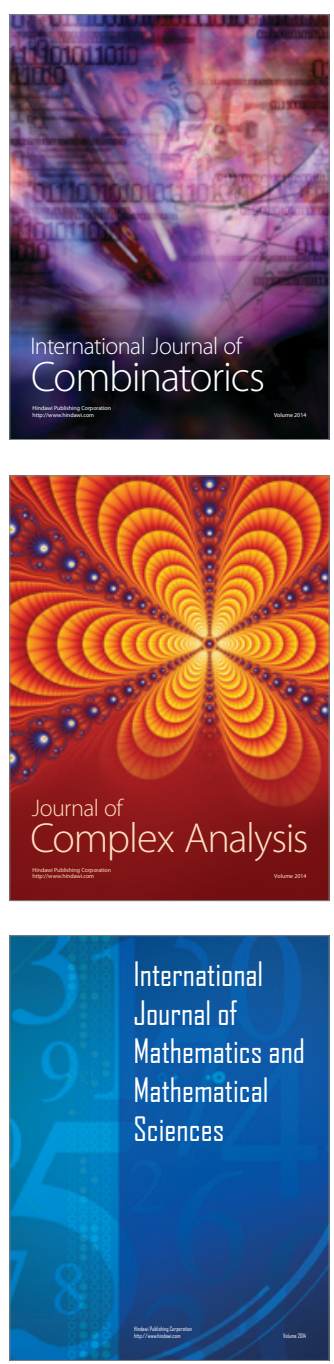
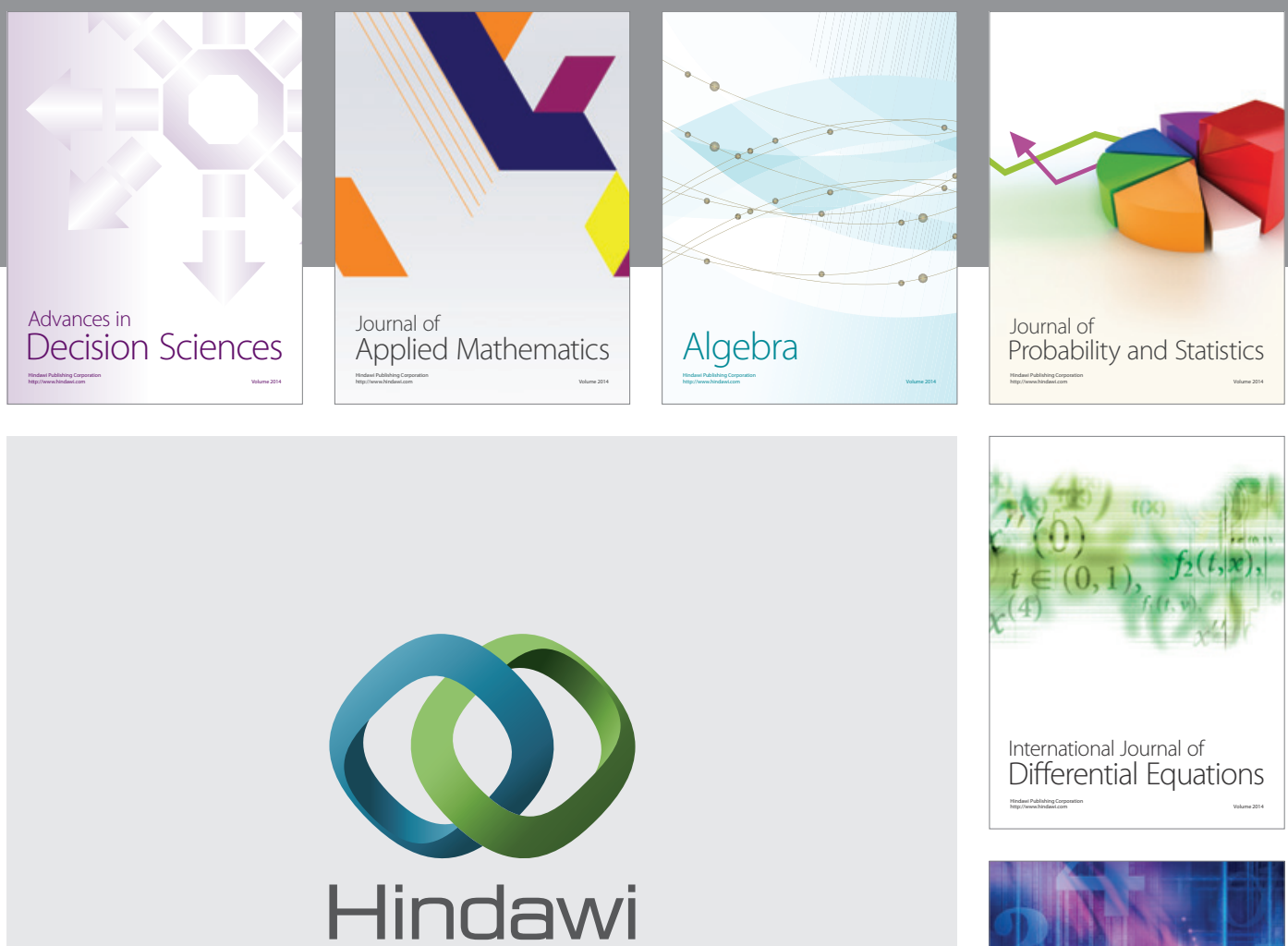

Submit your manuscripts at http://www.hindawi.com
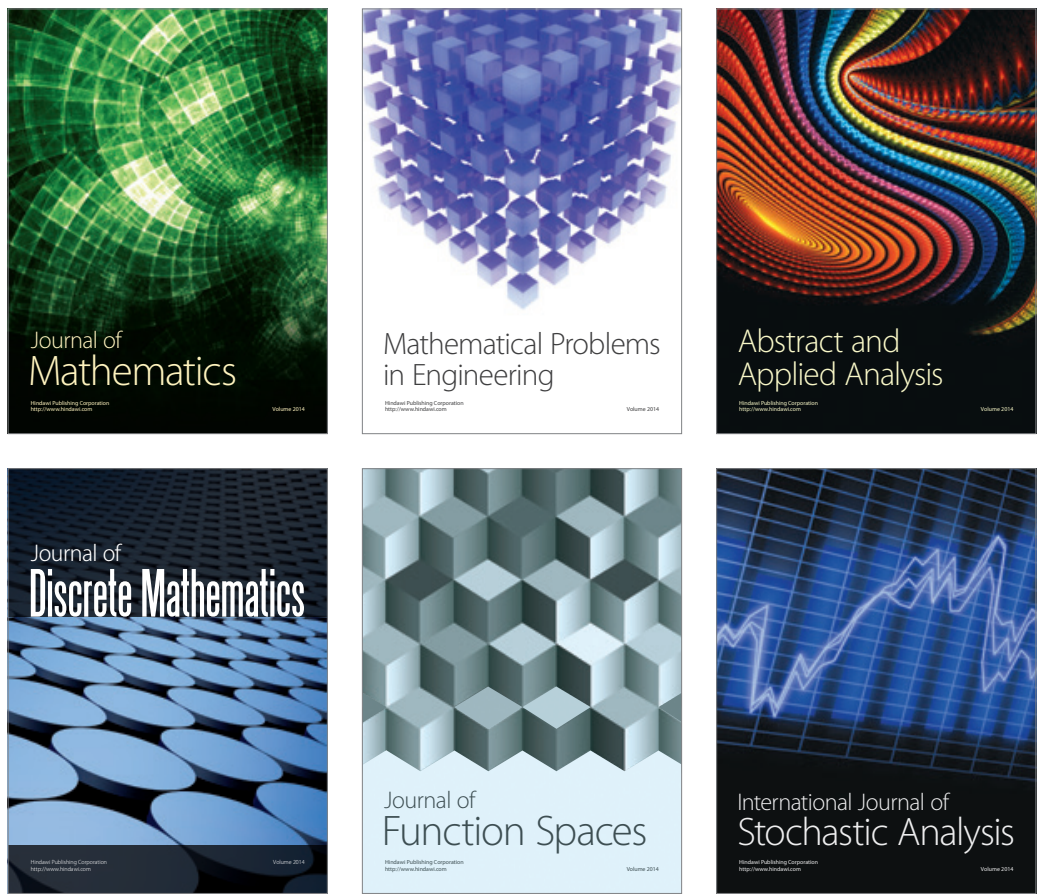

Journal of

Function Spaces

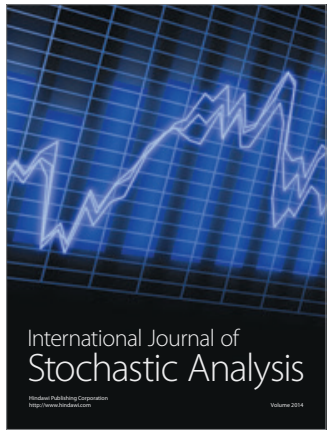

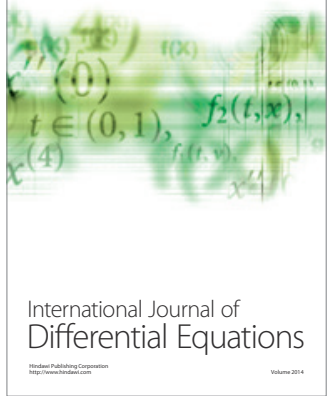
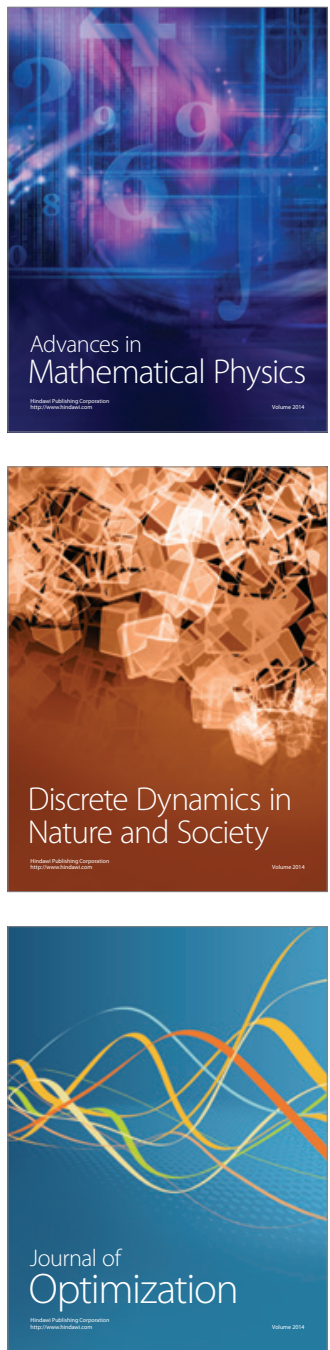